\title{
Movilización social en tiempos de recesión: un análisis de eventos de protesta en España, 2007-2015
}

\author{
Mobilization in times of recession: \\ an analysis of protest events in Spain, 2007-2015
}

\section{MARTÍN PORTOS}

Center on Social Movement Studies (COSMOS), Scuola Normale Superiore

Instituto Universitario Europeo

\section{Cómo citar/Citation}

Portos, M. (2016). Movilización social en tiempos de recesión: un análisis de eventos de protesta en España, 2007-2015. Revista Española de Ciencia Política, 41, 159-178. Doi: http://dx.doi.org/10.21308/recp.41.07

\section{Resumen}

Las movilizaciones del 15M han sido protagonistas de un ciclo más amplio de acciones colectivas contra la austeridad y el statu quo político en el contexto de la recesión. Esta contribución presenta y explora una base de datos original recabada con análisis de eventos de protesta para España entre 2007 y 2015, que permite capturar las fluctuaciones en el ámbito de la contienda política a lo largo de este período. En base a ella, se establece una periodización del ciclo de protestas y se analizan las dinámicas temporales de determinados aspectos relacionados con las mismas, como los asistentes, organizadores, niveles de disrupción, evolución de las demandas y distribución territorial.

Palabras clave: 15M, indignados, eventos de protesta, ciclo de acción colectiva, austeridad, España.

\begin{abstract}
$15 \mathrm{M}$ mobilizations represent the most relevant events of a broader cycle of collective actions against austerity and the political status quo in the context of recession. This contribution introduces and explores an original dataset collected through protest event analysis conducted for Spain between 2007 and 2015. It allows us to pin down the longitudinal fluctuations of political contention over this period. Based on it, a chronology of the cycle of protest is established; moreover, the temporal dynamics of some aspects related to protests are analyzed, such as protest participants, organizers, levels of disruption, evolution of claims and territorial distribution.
\end{abstract}

Keywords: 15M, indignados ('those outraged'), protest events, cycle of collective action, austerity, Spain. 
Los últimos años han estado marcados por elevados niveles de movilización y resistencia social en un contexto marcado por la crisis financiera global a lo largo y ancho del globo, desde Chile a Israel, pasando por Estados Unidos, Brasil, varios países árabes y el sur de Europa, entre otros muchos ejemplos.

Dentro del contexto español, la emergencia del $15 \mathrm{M}$ y el movimiento de los indignados en 2011 han alterado notablemente la escena de movilización socio-política. El 15 de mayo de 2011, el colectivo ¡Democracia Real Ya! reunió en el centro de Madrid a unos pocos cientos de manifestantes bajo los eslóganes «No nos representan» $\mathrm{y}$ «No somos mercancía en manos de políticos y banqueros». Esta manifestación tuvo lugar justo una semana antes de los comicios municipales y autonómicos (en 13 de las 17 comunidades autónomas). Tras la protesta, algunos jóvenes improvisaron una sentada pacífica en la plaza de Callao, que fue duramente reprimida. Como reacción, convergieron en la Puerta del Sol y, junto con cientos de simpatizantes adicionales informados gracias a medios digitales (\#SpanishRevolution se había convertido en trending topic en Twitter por aquel entonces), formaron una acampada, siguiendo el modus operandi de la plaza Tahrir, en El Cairo (Egipto). Las ocupaciones se extendieron a la mayoría de capitales de provincia españolas en menos de 24 horas.

De este modo, una campaña de protesta no partidista, organizada en asambleas públicas, abiertas y populares, emergía con comisiones específicas que facilitaban la participación directa ciudadana. Estas han supuesto las movilizaciones más intensas y concurridas desde la Transición al margen de instituciones intermedias de representación, como sindicatos y partidos políticos. No solamente más de un millón de personas han participado activamente, sino que más del $70 \%$ de españoles han mostrado actitudes positivas hacia las mismas (Martí i Puig, 2011). Este apoyo ha sido transversal, aunque los jóvenes de izquierdas altamente educados, interesados en política, con redes de contactos preestablecidas e insatisfechos con la democracia estuvieron sobrerrepresentados en las protestas, como demuestran diversos estudios empíricos existentes (Calvo et al., 2011; Calvo, 2013; Likki, 2012; Anduiza et al., 2014).

Tras unas semanas de ocupaciones, con la Puerta del Sol (Madrid) y la plaça de Catalunya (Barcelona) como puntos neurálgicos, el movimiento se disgregó en asambleas locales, que alimentaron el ciclo más amplio de acciones colectivas contra la austeridad. Así pues, limitar las actividades de protesta en el marco de la Gran Recesión al 15M y las ocupaciones en la Puerta del Sol es simplista y reduccionista. Nos impediría entender lo que en realidad ha sido un ciclo de confrontación social mucho más complejo e irregular, con variables estrategias, actores, interacciones y multitud de frentes abiertos en la acción colectiva contenciosa: antidesahucios, mareas, movimientos urbanos, etc.

No obstante, carecíamos de información sistemáticamente recabada que permitiese analizar y entender las recientes fluctuaciones temporales de las dinámicas de protesta en España. Esta nota, que supone una exploración preliminar de mi base de datos recabada con análisis de eventos de protesta, aspira a contribuir a llenar esta laguna. Esta técnica, Protest Event Analyisis (PEA), nace para dar cuenta de esta evolución longitudinal de las protestas, particularmente dentro de un período de 
conflicto intensificado y más allá de campañas específicas (Tarrow, 1989, 1991; Hutter, 2014).

Esta base de datos forma parte de un proyecto de investigación doctoral más amplio que trata de explicar las dinámicas temporales y de participación en el reciente ciclo de protesta español. En él se triangulan diversos tipos de datos empíricos y técnicas metodológicas (encuestas y métodos estadísticos, entrevistas, análisis de contenido, etc.) que ya han dado pie a varias contribuciones académicas. Por ejemplo, Portos (2016), con esta misma base de datos de PEA, establece una periodización del ciclo de protestas y explica la persistencia del pico de movilizaciones entre 2011 y 2013. Con datos de encuesta, Portos y Masullo (2016) estudian el impacto de la insatisfacción democrática para distintas intensidades de participación dentro del $15 \mathrm{M}$ con respecto a los no activistas. Por su parte, basándose en entrevistas con informadores clave, Fernández y Portos (2016) analizan la transición entre el abandono de estrategias no convencionales de acción colectiva en favor de vías institucionalizadas.

Esta nota de investigación tiene una doble aspiración. Por un lado, trata de presentar la primera base de datos que existe aplicando un PEA acerca del reciente ciclo de protesta español contra la austeridad y el statu quo político, con la excepción parcial de Calvo y Garciamarín (2016). Por otra parte, en relación con estos datos, se pretende analizar la evolución temporal de determinados aspectos relacionados con la contienda.

Esta nota se vertebra siguiendo estos dos ejes. A continuación, explicamos en qué consiste un análisis de eventos de protesta, en general, y exploramos las características más destacables de la base de datos específica que aquí se presenta. Después, exploramos las dinámicas evolutivas de algunos elementos durante el ciclo de protesta. Primero nos ocuparemos de las fases, eventos y participación; después de los niveles de disrupción y características organizativas, seguidos por las motivaciones principales y la distribución territorial. Finalizaremos subrayando las principales aportaciones de este breve informe y la base de datos.

PEA PARA ESPAÑA, 2007-2015: INTRODUCIENDO UNA NUEVA BASE DE DATOS

PEA es una técnica metodológica empleada para mapear, analizar e interpretar la ocurrencia de protestas a lo largo del tiempo y el espacio, y factores asociados con los mismos, a través de análisis de contenido (Koopmans y Rucht, 2002: 231; Hutter, 2014). Tradicionalmente, como en mi caso, se ha utilizado información recogida en periódicos (ej. Tarrow, 1989; Tilly, 1995; Kriesi et al., 1995; Beissinger, 2002). La unidad de análisis empleada son eventos, que he definido, siguiendo a Mark Beissinger (2002:14; Tilly, 2002), como «actos de contienda (colectiva) y potencialmente subversivos que canalizan prácticas normalizadas, modos o sistemas de autoridad».

En concreto, he ensamblado mi base de datos de PEA con datos procedentes de El País $(\mathrm{N}=2002)$. Me he servido de las versiones digitalizadas impresas almacena- 
das en El País Archivo ${ }^{1}$ y de su buscador de palabras clave ${ }^{2}$. Cada artículo viene con una serie de etiquetas agregadas. La herramienta de búsqueda automáticamente selecciona aquellos artículos que encajan con mi entrada introducida durante el lapso temporal escogido ${ }^{3}$. Basándose en estas etiquetas, los archivos de El País se estructuran en bloques temáticos. Como varios de estos bloques están asociados al comportamiento político no convencional en un contexto de austeridad y podrían contener eventos de protesta no codificados (p. ej., los titulados "protestas sociales», «actos de protesta», «manifestaciones», "huelgas», "conflictos laborales», «15M», etc.), estas áreas temáticas han sido manual y cuidadosamente revisadas entre los meses de enero de 2007 y febrero de 2015, ambos incluidos. La decisión final acerca de si un artículo en cuestión informa sobre un evento de protesta o no (ej. falsos positivos, referencias a eventos ya codificados, etc.) ha sido manualmente tomada para cada caso $^{4}$.

Asimismo, las -hasta 77- variables incluidas por evento de protesta registrado han sido codificadas manualmente, combinando elementos de los libros de códigos de Kriesi et al. (1995), el proyecto Dynamics of Collective Action ${ }^{5}$ y Beissinger (2002), amén de diversas innovaciones propias (p. ej., con respecto a la codificación del número de participantes y los niveles de disrupción, que se detallan más abajo). Así, esta base de datos de PEA para España contiene información detallada acerca del contexto del evento (lugar, fecha, duración), el tipo de protesta (p. ej., relacionada con la educación, nacionalismo, ecologista, vivienda, etc.), los organizadores, los repertorios de acción, nivel de disrupción y violencia, las proclamas y justificación, los objetivos y propósitos, los niveles de asistencia, la existencia de aliados, la introducción de reformas o concesiones inmediatas por parte de autoridades e instituciones.

No han sido empleadas técnicas de muestro diacrónico. Es decir, se ha comprobado $-\mathrm{y}$, en su caso, recogido- información para todos y cada uno de los días dentro de nuestra franja temporal (para el proceso de recolección de datos se emplearon 17 semanas consecutivas, con plena dedicación). Siguiendo a Kriesi et al. (1995), las secciones de opinión y editoriales han sido omitidas. Asimismo, se ha evitado seleccionar sobre la variable dependiente: se ha recabado información acerca de todos los tipos de

1. Hasta el 7 de febrero de 2012, cuando el departamento editorial cambió su sistema de funcionamiento. Desde entonces, los artículos impresos son idénticos a las versiones digitalizadas almacenadas en la Hemeroteca de El País.

2. Utilizar la versión impresa digitalizada permite tomar en consideración, además de la edición nacional de El País, todas sus versiones territoriales simultáneamente.

3. En la lista de palabras clave se encuentran: protesta, manifestación, escrache, 15M, indignados, marea, movilización, marcha, acampada, sentada, boicot.

4. Algunas palabras clave como corrupción, austeridad, asamblea y concentración han sido descartadas por la cantidad ingente de falsos positivos generados, que imposibilitaba gestionarlas manualmente.

5. Coordinado por D. McAdam, J. McCarthy, S. Olzak y S. Soule. Más información en http:// web.stanford.edu/group/collectiveaction/cgi-bin/drupal/ 
eventos de protesta encontrados, no solo los estrictamente relacionados con la austeridad, la crisis, asuntos laborales o el statu quo político.

Aunque PEA es una técnica longitudinal muy útil para analizar tendencias longitudinales de los actos de protesta, a menudo presenta dificultades que exigen cautela y cuidado en el manejo de este tipo de datos (para introducciones, revisiones y análisis de sus limitaciones, véanse Koopmans y Rucht, 2002; Earl et al., 2004; Hutter, 2014) ${ }^{6}$. El gran desafío reside en la selectividad de la información publicada por los medios de comunicación. Los eventos a los que se da cobertura «nunca son una muestra representativa (ni aleatoria)» (Della Porta, 2014: 451). Es decir, existe un claro sesgo en la selección de los eventos publicados y los medios solo se hacen eco de una fracción de los eventos realmente existentes (Earl et al., 2004; Hutter, 2014)7.

No obstante, los medios impresos han sido y siguen siendo una arena crucial para el «claim-making» (entendido como realización de demandas) ${ }^{8}$, ya que los actores, en un momento u otro, tienden a recurrir a los mismos para hacer sus visiones públicas (Earl et al., 2004). Algunos autores han argumentado que si estos eventos carecen de repercusión mediática y no llegan a su potencial audiencia quizás ni tan siquiera deban ser considerados «eventos de protesta» (Gamson y Wolfsfeld, 1993: 116).

El horizonte temporal del estudio está comprendido entre enero de 2007 y febrero de 2015. Para analizar la evolución de las dinámicas de protesta bajo la sombra de la austeridad en España, estudio el desarrollo de la contienda tras los primeros síntomas de la Gran Recesión en 2007 (cuando se observan los primeros cambios en las tendencias de desempleo juvenil, el pico de endeudamiento a nivel doméstico, el estallido de la crisis financiera global derivada de las hipotecas basura, etc.) y hasta finales de febrero de 2015. Este es un momento oportuno para finalizar nuestro período de observación por tres motivos principales.

Primero, por criterios de índole económica. Aunque muchos efectos de la crisis persisten más allá de esta fecha, varios macroindicadores apuntan hacia un cambio de tendencia en el ciclo económico. Por ejemplo, la prima de riesgo se encuentra por debajo de los cien puntos básicos por primera vez desde 2005 y el nivel de desempleo

6. Como el eminente Charles Tilly (2002: 249) había advertido: «Cualquiera que construye [una base de datos con eventos] se preocupa inevitablemente por su selectividad, fiabilidad, verificabilidad, comparabilidad, interconexión e inclusión. Y si los compiladores de catálogos de eventos no lo hacen, lo harán sus críticos».

7. A fin de minimizar potenciales limitaciones derivadas del uso de una única fuente primaria (sesgo de selectividad) e ideológicos, he realizado un test parcial con datos procedentes de $E l$ Mundo. He comparado dos meses aleatorios, pre y pos-15M (abril de 2009 y noviembre de 2013) y no he encontrado diferencias sustanciales en cuanto al nivel de eventos cubiertos (correlación superior al $85 \%$ ).

8. Una claim se refiere a «una unidad de la acción estratégica en la esfera pública [...] Es una pronunciación de la opinión política en forma de acción verbal o física independientemente de la forma de expresión (el debate, la protesta, la decisión del juez, etc.) y del tipo de actor (Gobierno, movimiento social, periodista, etc.)»(Koopmans, 2002). 
-aunque todavía muy elevado- se mantuvo durante tres trimestres consecutivos por debajo del $24 \%$, un hecho inaudito en el lustro precedente.

El segundo argumento se refiere a las propias dinámicas políticas. Principios de marzo de 2015 supone el comienzo de la campaña electoral para las elecciones autonómicas andaluzas. Son los primeros comicios en un año con un calendario electoral muy intenso y apretado (elecciones municipales y en trece autonomías en mayo, catalanas en septiembre y generales en diciembre). Implica que los medios de comunicación, la opinión pública y los esfuerzos organizativos y de movilización se focalizan hacia la dimensión electoral de la participación política, especialmente tras la emergencia de fuerzas institucionalizadas como Podemos o diversas alternativas municipales (p. ej., Barcelona en Comú, Ahora Madrid, Marea Atlántica, etc.)

Una tercera razón, tal y como apuntan nuestros datos, es que surge una fase de desmovilización paralelamente a la emergencia de estas nuevas fuerzas institucionalizadas, que supone un restablecimiento del orden social, la reducción drástica del número de eventos y el final del ciclo de protesta (Tarrow, 1989, 1991; Beissinger, 2002). No obstante, las consecuencias de la ola de protestas, de diversa índole (biográficas, culturales, políticas), pueden prolongarse en el tiempo (Giugni, 2008).

\section{FASES, EVENTOS Y PARTICIPACIÓN}

Al agregar nuestros datos de eventos mensualmente, el gráfico 1 nos arroja una imagen de la evolución de las protestas compleja, con una trayectoria caracterizada por grandes fluctuaciones. Valles profundos y picos agudos se observan para el período comprendido entre enero de 2007 y febrero de 2015. Este no es un aspecto novedoso, ya que redunda en hallazgos sólidos para contextos tan variopintos como el ciclo de conflictos industriales en la Europa Occidental de finales de los sesenta y los setenta (Crouch y Pizzorno, 1978) o las movilizaciones nacionalistas y el colapso de la URSS (Beissinger, 2002).

Ya que esta irregularidad dificulta discernir la tendencia de las series, en el gráfico 2 utilizamos medias móviles para suavizarlas (con 5 períodos) ${ }^{9}$. En líneas generales, existen pocos eventos durante el período 2007-2010, con movilizaciones masivas muy ocasionales. A pesar de algunos picos precedentes, un clímax de protestas tuvo lugar tras el desencadenante que ha supuesto el 15M, a mediados de 2011, y se extendió hasta bien entrado 2013. Posteriormente, las protestas decayeron progresivamente. Calvo y Garciamarín (2016) apuntan una tendencia similar.

9. Las técnicas de suavización son un recursos estándar y ampliamente utilizado en los trabajos con series temporales (ej. Accornero y Ramos Pinto, 2014). Las tendencias en mis series no son estacionales; la intensidad un pico no puede ser calculada en base al valor de picos previos. 
GrÁFICO 1.

Eventos de protesta en España, 01/2007- 02/2015. Datos brutos del PEA

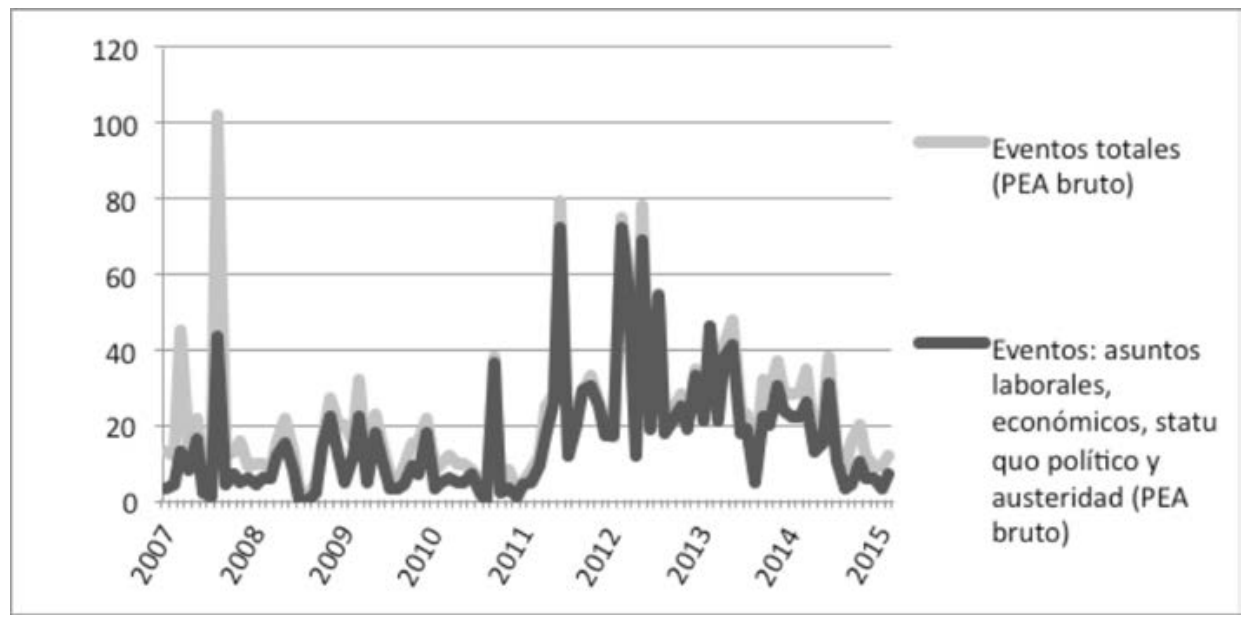

GrÁFICO 2.

Eventos de protesta en España, 01/2007- 02/2015. Media móvil (aplicando un filtro SUAVIZANTE A LOS DATOS BRUTOS DEL PEA, 5 PERÍODOS)

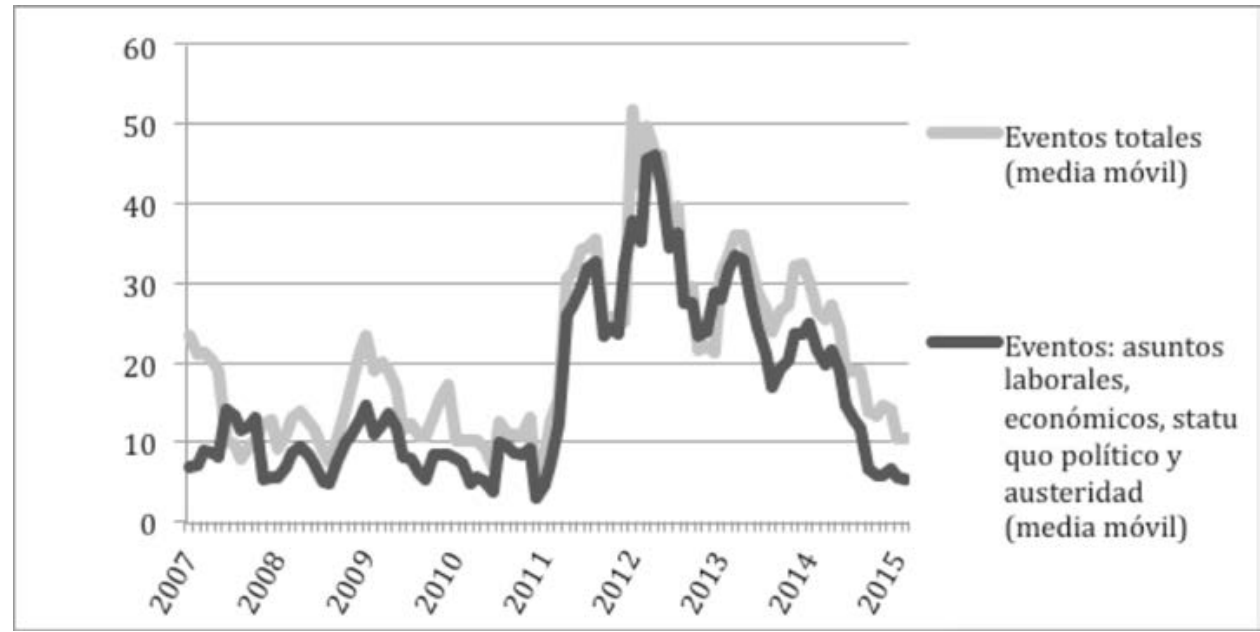

Para los dos gráficos, nivel de agregación: mensual. $\mathrm{Y}=$ número de eventos. $\mathrm{X}=$ tiempo (años, 2007-2015). Tono claro: todo tipo de eventos de protesta. Tono oscuro: solo eventos relacionados con el trabajo, asuntos económicos, statu quo político y austeridad.

Fuente: elaboración propia a partir de datos recabados de El País (N=2002). 
También podemos observar cómo la proporción de eventos contra la austeridad, la crisis, el statu quo político y asuntos laborales es alta con respecto al total del número de eventos -aproximadamente, dos tercios a lo largo del período de observación-. Esta proporción es especialmente elevada durante el clímax de las protestas, cuando se corresponden casi con el $90 \%$ de los eventos totales. En la fase inicial del ciclo, esta proporción es notablemente inferior.

Hemos agregado los eventos como reacción a agravios de índole socioeconómica (desigualdad, pobreza, desempleo, asuntos laborales) y política puesto que ambas crisis, particularmente en un contexto de recesión, no pueden ser consideradas aisladamente (Zamora-Kapoor y Coller, 2014). La respuesta institucional a los desafíos económicos subyace a las percepciones políticas ciudadanas. Es más, las reacciones contra el statu quo político se refieren no solo al mal funcionamiento de los mecanismos de representación democrática, la corrupción y mala praxis o la manifiesta incapacidad de las élites para satisfacer parte de las demandas ciudadanas -que también-, sino que atañen a la propia gestión de la crisis.

Un aspecto sensible con PEA es el cálculo y estimación del número de participantes en un evento determinado. Dependiendo de la fuente, los datos tienden a diferir sobremanera, son escasos y parciales. Para corregir este potencial sesgo, he recabado información sobre los datos de participación proporcionados por las tres fuentes principales por separado, cuando estaba disponible: 1) policía, autoridades o instituciones gubernamentales; 2) los organizadores; y 3 ) el propio periódico El País. Como las autoridades tienden a infraestimar el tamaño de las protestas y los organizadores lo sobreestiman, se utilizan coeficientes correctores a fin de calcular la desviación con respecto a la media para los casos donde dispongo de información completa acerca de los tres indicadores. Y he extrapolado esos coeficientes penalizados para cada una de las tres fuentes, que calculan la desviación media de las estimaciones, al total de la muestra a fin de obtener tres medidas de participación corregidas para cada fuente. Finalmente, he calculado un índice de participantes definitivo a partir de la media de las tres primeras fuentes (corregidas por sus respectivos coeficientes de penalización). Cuando se proporcionan solamente estimaciones de asistencia, con cifras vagas o no específicas (p. ej., «unas pocas decenas», «varios cientos», «muchos miles», etc.), se transforma en un indicador continuo $\left(\mathrm{N}^{\prime}=505\right)^{10}$.

10. Para la transformación en una variable continua, se siguen los criterios estipulados para Dynamics of Collective Action: 1) <100 participantes; 2) 100-999; 3) 1000-4999; 4) 5000-9999; 5) 10.000-19.999; 6) 20.000-49.999; 7) 50.000-99.999; 8) 100.000-199.999; 9) 200.000499.999 y 10) 500.000 . 
GRÁFICO 3.

Participantes en EVENTOS DE PROTESTA EN EspaÑa, 01/2007- 02/2015

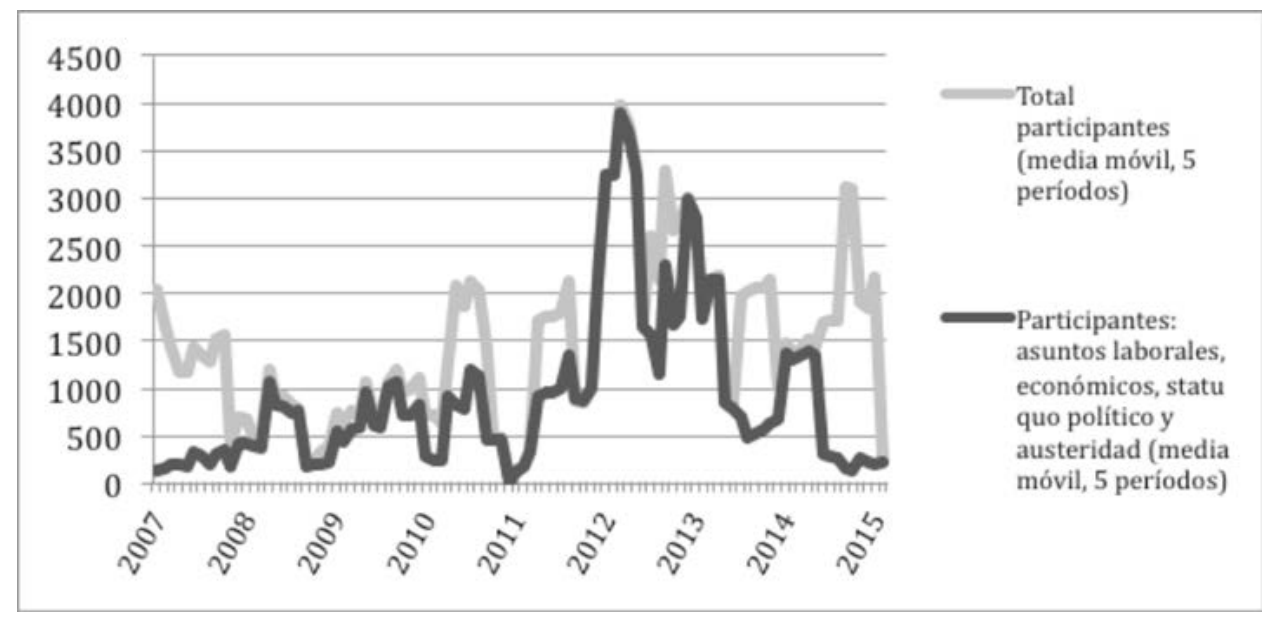

Nivel de agregación: mensual. $Y=$ número definitivo -corregido- de participantes en eventos de protesta. $\mathrm{X}=$ tiempo (años, 2007-2015). Medias móviles, aplicando un suavizador a los datos brutos del PEA (5 períodos). Tono claro: total de participantes en todos los tipos de eventos de protesta. Tono oscuro: solo eventos relacionados con el trabajo, asuntos económicos, statu quo político y austeridad.

Fuente: elaboración propia a partir de datos recabados de El País (N=2002) (Portos, 2016).

De acuerdo con las teorías clásicas de ciclos de acción colectiva (Tarrow, 1989, 1991; Tilly, 1995; Della Porta, 2014), podemos distinguir tres fases en una ola de protestas: ascenso, cima y descenso. Nuestro caso no presenta una excepción a esta regla si atendemos a la evolución estratégica y táctica de los actores en contienda.

Sabemos que inicialmente un ciclo escala sobre movimientos preexistentes y llega a capas más amplias de la población a medida que tiene recursos disponibles y las oportunidades políticas se abren. En esta fase, actores de la sociedad civil como sindicatos, partidos y organizaciones de movimientos sociales tradicionales jugaron un rol preeminente, simultáneamente apropiándose y ensanchando las oportunidades políticas, pues el ciclo asciende en un contexto donde se concatenan las crisis política y socio-económica anteriormente mencionadas.

El 15M emergió, por un lado, sobre el legado de las tres fases de movilización en España que destaca Viejo $\left.(2012)^{11}: 1\right)$ pro-democratización durante la Transición; 2) los nuevos movimientos sociales (p. ej., pacifista anti-OTAN), conflictos laborales y educativos de los ochenta, y 3 ) las movilizaciones del movimiento altermundialista en los noventa (Jiménez y Calle 2007; Flesher Fominaya 2014). Además, una red con múltiples capas de activistas fueron capaces de crear sus propios foros, espacios de encuentro y debate durante la parte baja de la ola (Portos, 2016). El surgimiento del

11. Para un recorrido histórico de la protesta en España en general, consúltese Cruz (2015). 
$15 \mathrm{M}$ no puede entenderse sin el sedimento proporcionado por al menos siete movimientos que, aunque con una capacidad de movilización y visibilidad limitadas, florecieron desde 2003 y ganaron vigor a finales de la primera década del 2000 (por una vivienda digna, okupas, estudiantes anti-Bolonia, la juventud precaria, contra el bipartidismo, por la cultura librelcomunes digitales y por la auto-determinación en Cataluña). Asimismo, nacida en el seno de la izquierda autónoma a finales de los noventa, la iniciativa ciudadana Rompamos el Silencio supone un «madrugador» y clave antecedente. Basada en la desobediencia civil, la acción directa no violenta y la organización horizontal, descentralizada y asamblearia, esta red aglutina a múltiples colectivos y vertebra reivindicaciones de diversa índole (feminista, anti-globalización, pacifista, etc.). Además, son destacables las sinergias proporcionadas por otras campañas de movilización durante esa época (p. ej., contra la guerra en Irak o el hundimiento del petrolero Prestige).

Con estos precedentes, el 15M surgió como una explosión de descontento con el statu quo político («politics-as-usual») en un escenario de acuciante y creciente privación material. Inicialmente impulsado por organizaciones como Juventud sin Futuro y ¡Democracia Real Ya!, el 15M y sus asambleas enseguida adoptaron repertorios y características organizacionales distintivos. El 15M supuso un primer desencadenante, uno de esos "momentos de locura colectiva [...] donde todo deviene posible» (Zolberg, 1972:183), que dio lugar a un pico agudo de movilizaciones que se prolongó en el tiempo, hasta bien entrado 2013.

El $15 \mathrm{M}$ ha persistido durante este período. De un movimiento general que aspiraba a cambiar el sistema socioeconómico, abandonó la ocupación visible de las plazas centrales y se descentralizó a través de asambleas locales. Aunque al regresar a sus raíces el $15 \mathrm{M}$ perdió visibilidad y participantes totales a corto plazo, la transformación permitió a los activistas retomar contacto con las necesidades más básicas de la ciudadanía, más allá de las prácticas idealistas y prefigurativas en las acampadas del 15M. Así, los indignados se especializaron y unieron sus esfuerzos a los movimientos de base en áreas diversas, de acuerdo con necesidades apremiantes. Por ejemplo, las actividades desarrolladas por el movimiento de lucha contra los desahucios, y particularmente la Plataforma de Afectados por la Hipoteca, se acentuaron durante los meses que siguieron el pico de protestas del 15M, ganando gran legitimidad popular ${ }^{12}$. Asimismo, diversas campañas, denominadas mareas, emergieron con el objetivo de luchar contra los recortes en sectores específicos, como la marea blanca para proteger la calidad del sistema de salud y contra medidas que conlleven su privatización parcial; la marea verde contra los recortes en educación pública o la marea naranja en defensa del trabajo social y las políticas de bienestar y dependencia, entre otras. A pesar de la fragmentación organizativa, en muchas de estas mareas y en las dos huelgas generales de 2012 se

12. De acuerdo con un sondeo de Metroscopia para El País, el apoyo popular a la campaña de escraches para luchar contra los desahucios rondaba el $80 \%$ en 2013 http://politica.elpais.com/ politica/2013/04/07/actualidad/1365358645_241274.html 
entretejieron alianzas entre actores asociados al 15M, sindicatos y otros colectivos claves para mantener a buena parte de la sociedad civil movilizada.

Todo ciclo, y este no ha sido una excepción, trae consigo nuevas demandas -más bien, reenfocadas y adaptadas-, repertorios de acción innovadores y se forjan identidades colectivas ${ }^{13}$. Si bien alcanzar estándares de movilización elevados es costoso, mantenerlos a lo largo del tiempo es, si cabe, todavía más difícil, ya que la "euforia inicial de la primavera de las gentes parece evaporarse rápidamente» (Tarrow, 2011:198). Aunque los movimientos y sus estrategias conllevaron diferentes niveles de éxito en el caso español (p. ej., se redujeron los desahucios, se paralizaron determinadas leyes, se atrajo hacia la arena política a buena parte de la juventud previamente despolitizada, etc.), la capacidad para perseguir aspiraciones adicionales a través de las mismas tácticas era limitada y las tácticas disuasorias empleadas por las autoridades cada vez más eficientes.

Sabemos que la desmovilización en un ciclo de acción colectiva habitualmente resulta de la concatenación de dos procesos: radicalización e institucionalización (Tarrow, 1989, 1991, 2011). A medida que se desarrolla el ciclo, nuevos agentes movilizadores emulan a los iniciadores y compiten por alcanzar a nuevos sectores de la población a través de campañas y estrategias más atractivas, normalmente de sectores claves a periféricos, siguiendo la lógica de una marea, como Tarrow (1989) apunta. Y esta re-intensificación del conflicto reduciría las audiencias a las que apela el movimiento, conllevaría cierta involución sectaria y conduciría a un cambio de aspiraciones: mientras algunos optan por rutas institucionalizadas, otros prefieren seguir adoptando formas de acción no convencionales, al tiempo que se radicalizan.

Si bien en el caso español la institucionalización fue pospuesta, ha tenido lugar desde finales de 2013, en paralelo a la desmovilización por vías no convencionales. La apuesta por la vía electoral es estratégica y deliberada, inicialmente impulsada por una élite reducida de activistas, pero abrazada por amplias bases, que son capaces de apropiarse de diversas oportunidades políticas florecientes: el agotamiento de los repertorios de protestas para alcanzar las aspiraciones de buena parte de los movimientos, junto con la crisis de los partidos mayoritarios y, particularmente, de los socialdemócratas, así como el aprovechamiento de la construcción simbólica de liderazgos (Fernández y Portos, 2016).

Sin embargo, como veremos a continuación, a pesar de varios fogonazos de violencia esporádica, no existe una clara tendencia hacia la radicalización en el ciclo de protesta español.

13. Uno de los rasgos definitorios de este ciclo, y particularmente del $15 \mathrm{M}$, ha sido la adopción de acampadas y asambleas como elementos organizativos y, simultáneamente, como formas de acción. Pero también se han incorporado y adaptado otras tácticas, como los escraches. Asimismo, se han desarrollado múltiples marcos estratégicos a lo largo del ciclo (p. ej., la juventud precaria, el 99\%, el pueblo contrapuesto a las élites corruptas y privilegiadas, etc.) en los que no podemos profundizar en esta nota por el límite de extensión establecido. 


\section{NIVELES DE DISRUPCIÓN}

Las dinámicas de contienda son el resultado de la interacción entre movimientos sociales y otros actores, varios de ellos estatales. En ocasiones, esta interacción se sitúa en el marco de la violencia y la represión, como apunta Jiménez (2005). Sin embargo, y aún siendo la violencia y la represión importantes para dirimir la trayectoria de ulteriores movilizaciones (Cruz, 2015), a tenor de nuestros datos, no se puede concluir que hayan existido fluctuaciones diacrónicas acusadas en los niveles de disrupción y violencia. No se ha incrementado el nivel de coerción de las autoridades empleado para reprimir las protestas ni los activistas han recurrido progresivamente a tácticas violentas a lo largo del tiempo y, contrariamente a lo que se ha argumentado desde las teorías clásicas de ciclos de contienda (Tarrow, 1989, 1991), no ha habido una radicalización en la fase de desmovilización (Portos, 2016). En esta línea, la inadecuación de las teorías de ciclos existentes para explicar los procesos de desmovilización ya ha sido subrayada por Della Porta (2014) en algunas olas de protesta previas a la consolidación democrática de Europa del Este y Latinoamérica a finales del siglo XX.

El gráfico 4 muestra, por un lado, la evolución de la represión policial medida con un indicador binario ( 1 si los activistas han utilizado estrategias violentas; 0 en caso contrario, o si no se registra el uso de violencia). Por otra parte, también se toma en consideración el grado de coerción (las medidas tomadas por las autoridades para reprimir las acciones de los activistas) en una escala $0-3^{14}$. Por último, también se captura la severidad e intensidad del desorden general, es decir, el nivel de violencia total. Se consigue combinando en una misma escala el grado de disrupción de las tácticas y recursos empleados tanto por activistas como por autoridades. Este indicador se toma prestado del estudio de Spilerman (1976), utilizado originariamente para capturar el nivel de daño humano y sobre la propiedad infligido en los conflictos raciales en Estados Unidos durante los sesenta, y se adapta a nuestro contexto específico. Su rango es $0-4^{15}$.

14. La codificación de las categorías es la siguiente: 0 = sin coerción conocida; 1 = coerción de baja intensidad (arrestos esporádicos y/o heridas, definidos como menos de 10); 2 = coerción substancial (definida como 10-75 arrestos o 10-40 heridos); 3 = gran represión (más de 75 arrestos o más de 40 heridos).

15. Se adjudica al evento la categoría máxima de disrupción posible, siempre y cuando satisfaga al menos dos de las condiciones especificadas a continuación: $0=$ sin violencia de ningún tipo conocida; 1 = baja intensidad de lanzamiento de objetos contundentes (p. ej., piedras, botellas), poco daño a la propiedad, $<15$ arrestos, $<8$ heridos; 2 = lanzamiento de objetos contundentes, enfrentamientos violentos, incendio provocado, pillaje, 10-30 arrestos, 5-15 heridos; 3 = violencia sustancial, incendio provocado, pillaje, destrucción considerable de la propiedad (valorada en 1.000-10.000 €), 25-75 arrestos, 10-40 heridos; 4 = violencia de gran intensidad; derramamiento de sangre, gran destrucción de la propiedad (valorada en $>10.000 €$ ), $>65$ arrestos, $>35$ heridos. 
GrÁFICO 4.

EVOlución de REPRESIÓN y VIOLENCIA EN EL TOTAL de EVENTOS DE PROTESTA ${ }^{16}$ EN España, DENTRO DEL CONTEXTO de LA GRAN RECESión, 01/2007-02/2015

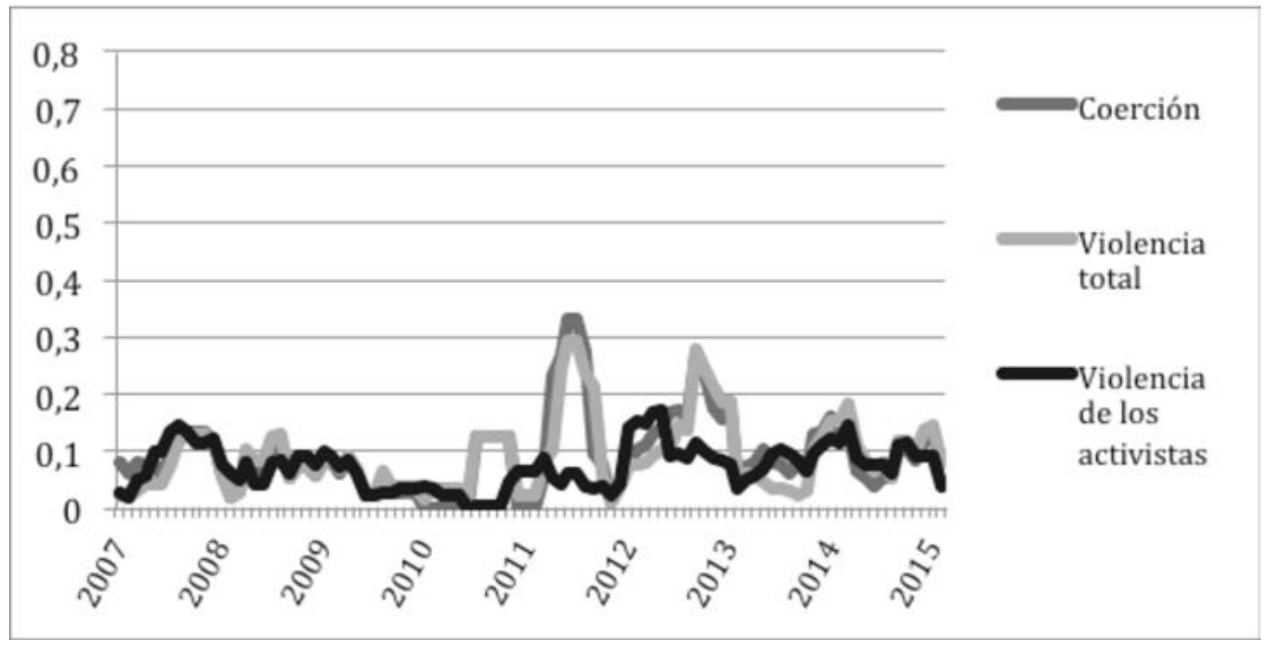

Nivel de agregación: mensual. $\mathrm{X}=$ tiempo (meses, 01/2007-02/2015). Tono medio: «coerción» se refiere al tipo de tácticas utilizado por las autoridades contra los activistas (escala 0-3). Tono claro: la «violencia total» captura de severidad del desorden general (escala 0-4). Tono oscuro-: la «violencia de los activistas» recoge si estos han utilizado algún tipo de violencia (escala $0-1$ ).

Fuente: elaboración propia a partir de datos procedentes del PEA con información de El País (N=2002) (Portos, 2016).

Como hemos apuntado, no ha existido una mayor coerción, al menos pública y visible, en lo que a mecanismos tradicionales de represión se refiere. Ello no contraviene la posibilidad de que las autoridades puedan haber aumentado su eficiencia y capacidad disuasoria a través del empleo de otro tipo de mecanismos coercitivos más subrepticios, como la proliferación de multas, tal y como apunta Martín García (2014). Tampoco ha existido una clara radicalización de los protestantes. De hecho, los picos de disrupción coinciden con los de protesta y las series siguen trayectorias similares (gráficos 1,2 y 3 ) -y no siguen una tendencia opuesta, sino paralela, a los eventos y participantes en la fase de desmovilización (también decrecen)-. Los picos de violencia se refieren, entre otros sucesos, al asalto al Parlamento catalán en junio de 2011, a la campaña "Rodea el Congreso» y varias movilizaciones antiausteridad en septiembre de 2012 y a los enfrentamientos vecinales en el barrio de Gamonal ante el plan de construcción de un bulevar (Burgos, enero de 2014).

Como se he apuntado en otras publicaciones (Portos, 2016), es muy probable que esta no radicalización esté vinculada a la necesidad de tender lazos y estrategias por

16. Los resultados para la submuestra de eventos contra el statu quo político, asuntos laborales, la crisis y la austeridad no cambian sustantivamente (gráfico no aportado). 
parte de las nuevas organizaciones con actores tradicionales, particularmente los sindicatos.

\section{CARACTERÍSTICAS ORGANIZATIVAS}

Durante este ciclo ha existido bastante tensión entre las nuevas organizaciones que emergieron en el contexto de la austeridad (p. ej., Juventud sin Futuro, asambleas del $15 \mathrm{M}$, etc.) y los sindicatos mayoritarios (p. ej., CC. OO., UGT, etc.). Sin embargo, el entendimiento mutuo puntual entre viejos y nuevos actores responde al interés de ambos. Por un lado, ante la crítica y el enorme respaldo social de nuevos actores, los sindicatos son conscientes de que pierden el liderazgo en la movilización y corren el riesgo de desvincularse definitivamente de las demandas de una sociedad cada vez más precarizada. Por el contrario, las nuevas organizaciones ven a los sindicatos como integrantes del statu quo político, alejados de las necesidades de la ciudadanía. No obstante, estos siguen teniendo una serie de recursos (materiales, experiencia, redes, acceso a instituciones, etc.) de las que carecen las nuevas organizaciones.

Es más, siguen teniendo el músculo para movilizar grandes capas de la ciudadanía. Por ejemplo, estas alianzas entre nuevos y viejos actores han sido cruciales para el éxito de movilización en campañas de protesta específicas, como las mareas blancas, las mareas verdes o las propias huelgas generales de 2012. Las nuevas organizaciones necesitan recurrir ocasionalmente a actos masivos intensos - «eventful performances» (Sewell, 1996) - o "ceremonias regulares de protesta» (en palabras de Casquete, 2005) para mantener el sentido e identidades colectivas, las diversas coaliciones unidas y motivadas, propagar marcos estratégicos e incrementar su capacidad de presión ante actores institucionalizados.

Una de las características más destacables de este ciclo es que han surgido nuevos actores con potencial para movilizar a gran cantidad de gente al margen de los partidos y sindicatos tradicionales. No obstante, estos últimos han jugado -y siguen haciéndoloun rol muy relevante en el ámbito de la protesta durante la Gran Recesión, como el gráfico 5 demuestra.

Algo muy similar ha acontecido en Portugal, tal y como Accornero y Ramos Pinto (2014) han subrayado recientemente. Y es que en España, este ciclo no ha supuesto la muerte de los sindicatos mayoritarios, sino que les ha forzado a adaptarse a un contexto social cambiante, con nuevos competidores. A pesar de las relaciones tensas entre sindicatos tradicionales y recientes organizaciones civiles, los actores nuevos y tradicionales han sido capaces de entretejer alianzas estratégicas -más o menos formales y con desigual permanencia en el tiempo. 
GRÁFICO 5.

PARTICIPANTES y ORGANIZADORES EN EVENTOS DE PROTESTA EN EsPAÑA, 01/2007- 02/2015

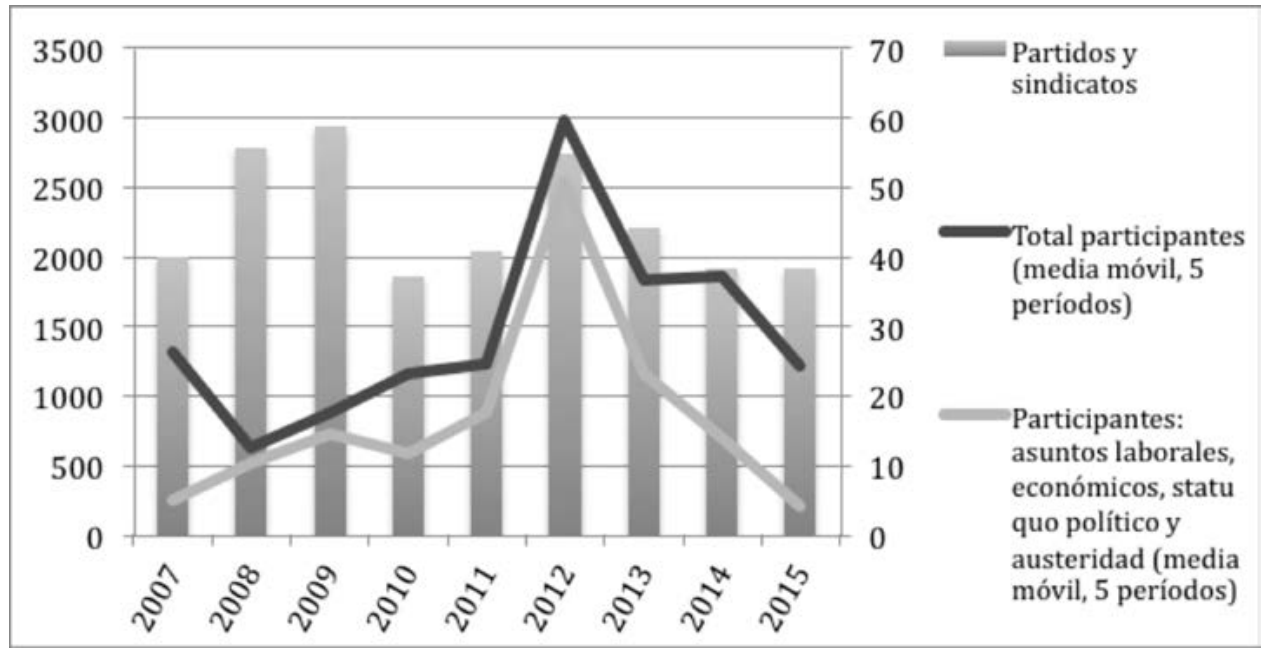

Nivel de agregación: anual (media mensual). Eje Y izquierdo= número -corregido- de participantes en eventos de protesta medido en millares (promedio anual, prorrateado mensualmente; variable continua).Se han utilizado medias móviles, aplicando un suavizador a los datos brutos del PEA, 5 períodos. Eje Y derecho= porcentaje de eventos organizados por partidos y/o sindicatos. $\mathrm{X}=$ tiempo (años, 2007-2015). Barras: organizadores tradicionales en proporción al total de eventos (suma de principales sindicatos y partidos). Línea oscura: total de participantes en todos los tipos de eventos de protesta. Línea clara: solo eventos relacionados con el trabajo, asuntos económicos, statu quo político y austeridad.

Fuente: elaboración propia a partir de datos recabados de El País $(\mathrm{N}=2002)$.

\section{MOTIVACIONES}

Nuestra base de datos de PEA también ayuda a arrojar luz sobre las motivaciones que subyacen a las dinámicas de protesta a lo largo del ciclo. Durante el punto álgido, entre mediados de 2011 y hasta finales de 2013, más del $70 \%$ de los eventos de protesta estaban directamente relacionadas con los recortes, la austeridad y la situación económica. Contrasta con la proporción de eventos motivados por estos factores en los primeros y últimos años del ciclo, inferior al $50 \%$. Así como cae el número de eventos y participantes totales, el peso relativo de las protestas directamente relacionadas con la crisis, recortes y austeridad hacen lo propio. Las movilizaciones asociadas con el empleo y desempleo, por un lado, y la corrupción, críticas al statu quo político y la implementación de decisiones políticas específicas, por otro, reflejan esta misma trayectoria decreciente durante la fase de desmovilización. En contraste, cerca del $30 \%$ de las protestas en 2011 estuvieron motivadas por este último grupo de factores, cuyo peso relativo era inferior al $10 \%$ antes de 2010. 
Gráfico 6.

PROBABILIDAD DE OCURRENCIA DE UN TIPO DE EVENTO DE PROTESTA

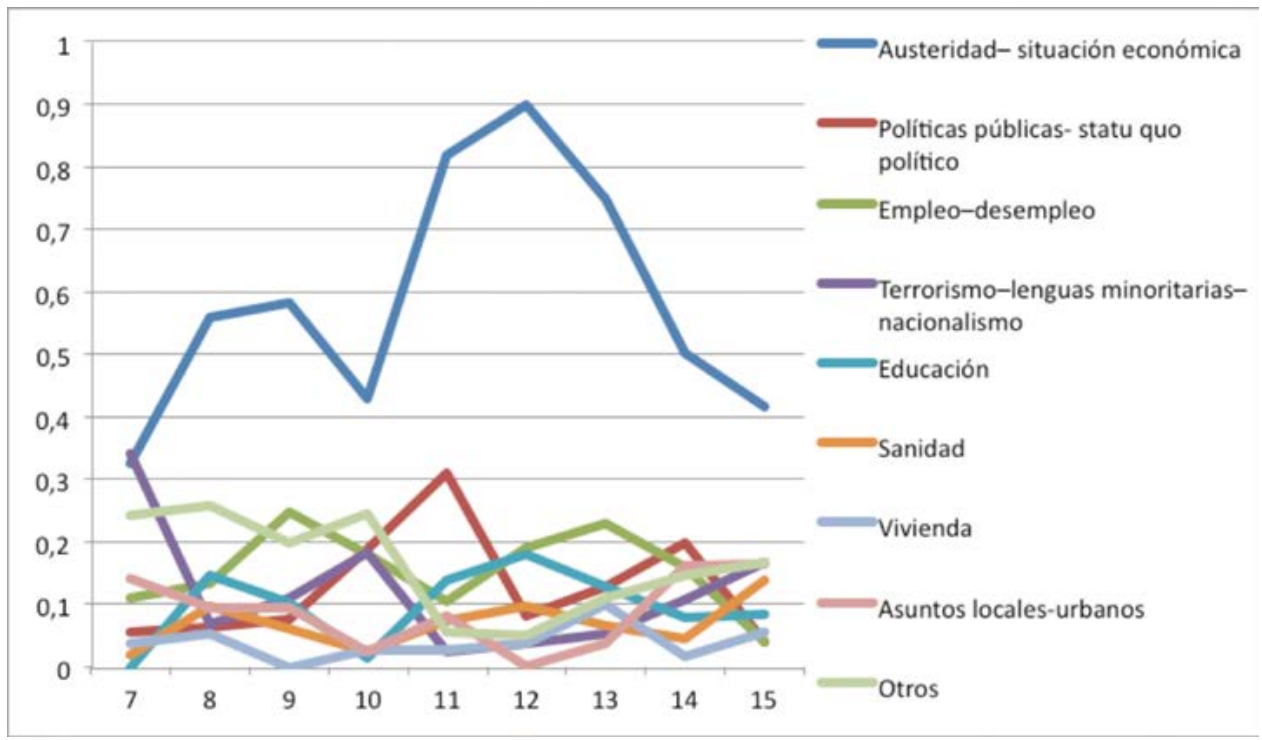

El «tipo» de evento se determina de acuerdo con el tema las principales demandas hechas públicas durante el mismo. Respuesta múltiple permitida, por lo que la probabilidad agregada en determinados puntos temporales excede 1 . Y = probabilidad 0-1. X = tiempo (años, 01/2007-02/2015). Nivel de agregación: anual.

Fuente: elaboración propia a partir de datos procedentes del PEA con información de El País (N=2002) (Portos, 2016).

* Véase el gráfico en color en la versión electrónico de la revista: http://recyt.fecyt.es/index.php/recp.

\section{DISTRIBUCIÓN TERRITORIAL}

Un último aspecto a considerar en esta nota de investigación concierne a la distribución geográfica de las protestas. Más de la mitad de los eventos en este ciclo han tenido lugar en Madrid y/o Cataluña. Se ofrecen datos agregados para todo el período, pues no existen tendencias diacrónicas destacables. Solo el 5\% de los eventos en la base de datos de PEA han tenido lugar fuera de las seis autonomías más subversivas, que aglutinan al $70 \%$ de la población total española. 
GrÁFICO 7.

EVENTOS DE PROTESTA POR COMUNIDAD AUTÓNOMA

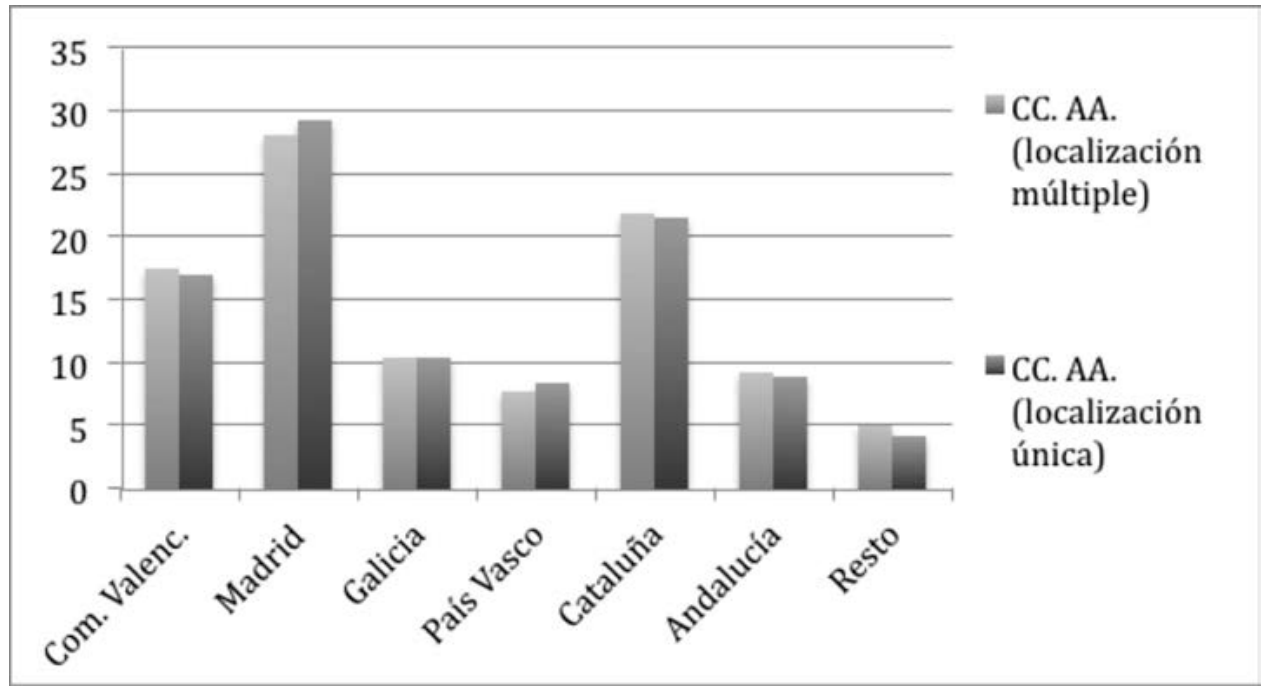

Tono claro: se permite localización múltiple (hasta 3 CC. AA. codificadas por evento; $\mathrm{N}=2002$ ). Tono oscuro: eventos con localización múltiple excluidos $\left(\mathrm{N}^{\prime}=1570\right)$. Y=porcentaje. X=CC. AA. Nivel de agregación: todo el ciclo, 01/2007$02 / 2015$.

Fuente: elaboración propia a partir de datos procedentes del PEA con información de El País.

\section{CONCLUSIONES}

En esta breve nota se presenta una base de datos recabada con análisis de eventos de protesta sobre España, 2007-2015. Esta técnica metodológica, desarrollada de la mano del paradigma de proceso político, se basa en análisis de contenido cuantitativo. Sirve para trazar la ocurrencia de protestas a lo largo del tiempo y el espacio. En la flexibilidad radica la gran virtud de PEA, pero también su principal limitación: es muy sensible ante determinadas decisiones tomadas durante la investigación, como por ejemplo, la selección de la unidad de análisis o el proceso de codificación. Aunque presente múltiples limitaciones (es costosa en términos de tiempo, los sesgos mediáticos son difíciles de controlar, etc.), PEA es útil para analizar dinámicas y tendencias del ciclo de protesta.

Esta evidencia empírica permite respaldar la interpretación del conjunto de movilizaciones acaecidas en España en el contexto de la recesión como un ciclo de protesta. Considerando los niveles de eventos y participantes, observamos que el ciclo consta de las tres fases tradicionales: ascenso, clímax y declive. El 15M supone el desencadenante de un pico de protestas que se mantiene hasta bien entrado 2013, cuando comienza la desmovilización. Si bien existe una evolución táctica hacia vías más institucionalizadas, no constatamos sin embargo una tendencia hacia la radicalización ni mayores niveles de disrupción durante la fase de desmovilización. 
Con esta base de datos, también podemos observar que los actores tradicionales (como partidos y sindicatos) siguen desempeñando un rol fundamental en la organización de protestas. Además, los niveles relativos de acciones relacionadas con la austeridad, el desempleo, la crisis y el statu quo político han sido variables, pero más acentuados durante los picos de protesta. Por su parte, la distribución territorial de las movilizaciones no ha sido equilibrada.

\section{Referencias}

Accornero, Guya y Pedro Ramos Pinto. 2014. «Mild Mannered”? Protest and Mobilisation in Portugal under Austerity, 2010-2013", West European Politics, 38 (3): 491-515. Disponible en: http://dx.doi.org/ 10.1080/01402382.2014.937587.

Anduiza, Eva, Irene Martín y Araceli Mateos. 2014. «Las consecuencias electorales del $15 \mathrm{M}$ en las elecciones generales de 2011", en Eva Anduiza, Agustí Bosch, Lluís Orriols y Guillem Rico (eds.), Elecciones generales 2011. Madrid: CIS.

Beissinger, Mark R. 2002. Nationalist Mobilization and the Collapse of the Soviet State. Cambridge: Cambridge University Press. Disponible en: http://dx.doi.org/10.1017/ CBO9780511613593.

Calvo, Kerman. 2013. «Fighting for a Voice: The Spanish 15M/Indignados Movement», en Cristina Flesher Fominaya y Laurence Cox (eds.), Understanding European Movements: New Social Movements, Global Justice struggles, Anti-Austerity Protest. Londres: Routledge.

Calvo, Kerman y Hugo Garciamarín. 2016. ¿Qué ha pasado con la movilización social? Continuidad y cambios en la protesta social en España. Informe 2016/28. Madrid: Fundación Alternativas Laboratorio. Disponible en: http://www.fundacionalternativas. org/public/storage/publicaciones_archivos/ce0bc292d3691a22338f26802e04b310. pdf.

Calvo, Kerman, Teresa Gómez-Pastrana y Luis Mena. 2011. «Movimiento 15M: ¿quiénes son y qué reivindican?», Zoom Político, 4: 4-17.

Casquete, Jesús. 2005. «Manifestaciones e identidad colectiva», Revista Internacional de Sociologia, 42: 101-125. Disponible en: http://dx.doi.org/10.3989/ris.2005.i42.198.

Crouch, Colin y Alessandro Pizzorno (eds). 1978. The resurgence of class conflict in Western Europe since 1968. London: MacMillan. Disponible en: http://dx.doi. org/10.1007/978-1-349-03022-4 y http://dx.doi.org/10.1007/978-1-349-03025-5

Cruz, Rafael. 2015. Protestar en España, 1900-2013. Madrid: Alianza Editorial.

Della Porta, Donatella. 2005. «Protest on unemployment: forms and opportunities», en Marco Giugni y Paul Statham (eds.), The Contentious Politics of Unemployment in Europe. Political Claim-making, Policy Deliberation and Exclusion from the Labour Market. Disponible en: http://www.eurpolcom.eu/exhibits/ch9-protest.pdf. [Consulta: 20 de febrero de 2013].

Della Porta, Donatella. 2014. "Cycles of protest and the Consolidation of Democracy», Partecipazione e Conflitto, 7 (3): 447-468. 
Earl, Jennifer, Andrew Martin, John D. McCarthy y Sarah A. Soule. 2004. «The use of newspaper data in the study of collective action", Annual Review Of Sociology, 30: 65- 80. Disponible en: http://dx.doi.org/10.1146/annurev.soc.30.012703. 110603.

Fernández, Joseba y Martín Portos (2016) «Moving from the squares to the ballot box. Podemos and the institutionalization of anti-austerity protests: a cycle-based approach», próximamente.

Flesher Fominaya, Cristina. 2014. «Debunking Spontaneity: Spain’s 15M/Indignados as Autonomous Movement», Social Movement Studies, 14 (2): 142-163. Disponible en: http://dx.doi.org/ 10.1080/14742837.2014.945075.

Gamson, William A. y Gadi Wolfsfeld. 1993. "Movements and media as interacting systems", Annals of the American Academy of Political Science, 528: 114-125. Disponible en: http://dx.doi.org/10.1177/0002716293528001009.

Giugni, Marco. 2008. «Political, Biographical, and Cultural Consequences of Social Movements», Sociology Compass, 2 (5): 1582-1600. Disponible en: http://dx.doi. org/10.1111/j.1751-9020.2008.00152.x.

Hutter, Swen. 2014. "Protest Event Analysis and its Offspring», en Donatella della Porta (ed.), Methodological Practices in Social Movement Research, Oxford: Oxford University Press. Disponible en: http://dx.doi.org/10.1093/acprof:oso/9780198 719571.003 .0014$.

Jiménez, Manuel. 2005. El impacto político de los movimientos sociales. Un estudio de la protesta ambiental en España. Madrid: CIS.

Jiménez, Manuel y Ángel Calle. 2007. «The Global Justice Movements in Spain», en D. Della Porta (ed.), The Global Justice Movement: Cross-National and Transnational Differences. Boulder: Paradigm.

Koopmans, Ruud. 2002. "Codebook for the analysis of political mobilisation and communication in European public spheres». Disponible en: http://europub.wzb. eu/codebooks.en.htm.

Koopmans, Ruud y Dieter Rucht. 2002. «Protest Event Analysis», en Bert Klandermans y Suzanne Staggenborg (eds.), Methods of Social Movement Research. Minneapolis, MN: University of Minnesota Press.

Kriesi, Hanspeter; Ruud Koopmans, Jan Willem Dyvendak y Marco Giugni. 1995. New Social Movements in Western Europe: A Comparative Analysis. London: UCL Press.

Likki, Tiina. 2012. «15M Revisited: A Diverse Movement United for Change», Zoom Político, 11: 1-16.

Martí i Puig, Salvador. 2011. «15M: The Indignados», en J. Bryne (ed.), The Ocuppy Handbook. New York: Back Bay Books.

Martín García, Óscar. 2014. «Soft repression and the current wave of social mobilizations in Spain», Social Movement Studies, 13 (2): 303-308. Disponible en: http:// dx.doi.org/10.1080/14742837.2013.863147.

Masullo, J. y Martín Portos. 2015. «Voicing outrage unevenly. Democratic dissatisfaction and differential participation in the Spanish $15 \mathrm{M}$ protest campaign». Sin publicar.

Portos, Martín. 2016. "Taking to the streets in the context of austerity: a chronology of the cycle of protest in Spain, 2007-2015", Partecipazione e Conflitto, 9 (1): 181-210. 
Sewell, William H. 1996 «Three Temporalities: Toward an Eventful Sociology», en Terrence J. McDonald (ed.), The Historic Turn in the Human Sciences. Ann Arbor, MI: University of Michigan Press.

Spilerman, Seymour. 1976. «Structural characteristics of cities and the severity of racial disorders", American Sociological Review, 41 (5): 771-793. Disponible en: http://dx.doi.org/10.2307/2094726.

Tarrow, Sidney G. 1989. Democracy and Disorder: Protest and Politics in Italy, 19651975. Oxford: Oxford University Press.

Tarrow, Sidney G. 1991. "Ciclos de protesta», Zona Abierta, 56: 53-76.

Tarrow, Sidney G. 2011. Power in Movement. $3^{a}$ ed. Cambridge: Cambridge University Press.

Tilly, Charles. 1995. Popular Contention in Great Britain, 1758-1834. Cambridge, MA: Harvard University Press.

Tilly, Charles. 2002. "Event catalogs as theories», Sociological Theory, 20 (2): 248254. Disponible en: http://dx.doi.org/10.1111/1467-9558.00161.

Viejo, Raimundo. 2012. «Indignación: Política de movimiento, nueva ola de movilizaciones y crisis de representación», en Fermín E. Rivas, M. Ángeles Mascott y Efrén Arellano (eds.), La actuación del legislativo en los tiempos de crisis. México: CESOP, pp. 123-156.

Zamora-Kapoor y Xavier Coller. 2014. "The Effects of the Crisis: Why Southern Europe?», American Behavioral Scientist, 58 (12): 1511-1516. Disponible en: http://dx.doi.org/10.1177/0002764214530649.

Zolberg, Aristide R. 1972. "Moments of Madness", Politics and Society, 2: 183-207. Disponible en: http://dx.doi.org/10.1177/003232927200200203.

Presentado para evaluación: 6 de noviembre de 2015.

Aceptado para publicación: 3 de abril de 2016.

\section{MARTÍN PORTOS}

martin.portos@eui.eu

Investigador doctoral Salvador de Madariaga en el Instituto Universitario Europeo (IUE) de Florencia y research fellow en el Center of Social Movement Studies (COSMOS), Scuola Normale Superiore. Trabaja principalmente sobre protestas antiausteridad en el sur de Europa. Se graduó en Ciencia Política y de la Administración por la Universidade de Santiago de Compostela (Premio Autonómico y Primer Premio Nacional de Fin de Carrera). Ha completado un MSc Politics Research (Comparative Government) por la Universidad de Oxford y un MRes Political and Social Sciences por el IUE. Ha obtenido becas y ayudas, entre otras, de la Fundación Caja Madrid, Obra Social La Caixa, Linares Rivas Universidad de Oxford, Centro de Investigaciones Sociológicas y IUE-Ministerio de Educación, Cultura y Deporte. 